\title{
Polymorphism at Codon 72 of p53 Is Not Associated with Cervical Cancer Risk
}

Elsa K. Malcolm, M.D., Gwen B. Baber, James C. Boyd, M.D., Mark H. Stoler, M.D.

Department of Pathology (EKM, GBB, JCB) and Division of Surgical and Cytopathology (MHS), University of Virginia Health Sciences Center, Charlottesville, Virginia

P53 allelic polymorphism at codon 72 has been studied as a possible predisposing factor for cervical carcinogenesis with inconsistent results. Storey and colleagues recently published the interesting finding of a 7 -fold increased risk for cervical cancer in women homozygous for the arginine allele at codon 72. This stimulated a number of independent investigations, the majority of which found no association of cervical cancer and arginine homozygosity.

With the use of a modified Storey method for determining codon 72 allelotypes, DNA was examined from 431 microdissected, formalin-fixed, archival cervical conization specimens ranging from low-grade squamous lesions to invasive cancer. An alternative independent method using restriction fragment length polymorphism analysis was performed on all arginine homozygotes and all indeterminate cases for confirmation and final allelotype assignment.

With the use of Storey's method alone, logistic regression suggested an association (odds ratio, 1.42) between arginine homozygosity and invasive disease. However, with the use of the combined method for accurate allelotyping, this trend disappeared (odds ratio, 1.00), the discordance was clearly resolvable as being due to methodologic variables.

With the use of two separate methods for codon 72 allelotyping and accounting for a number of the issues raised in previously published reports, there is no increased risk for invasive cervical cancer associated with arginine homozygosity at codon 72 of p53.

Copyright () 2000 by The United States and Canadian Academy of Pathology, Inc.

VOL. 13, NO. 4, P. 373, 2000 Printed in the U.S.A.

Date of acceptance: August 24, 1999.

Address reprint requests to: Mark H. Stoler, M.D., Division of Surgical and Cytopathology, Box 214, University of Virginia Health Sciences Center, Charlottesville, VA 22908; e-mail: mhs2e@virginia.edu; fax: 804-924-0217.
KEY WORDS: Cervix, Neoplasia, p53, Papillomavirus, Polymorphism.

Mod Pathol 2000;13(4):373-378

Human papillomaviruses (HPVs) are epitheliotropic double-stranded DNA viruses that have been implicated in cervical carcinogenesis. More than 100 HPV genotypes have been identified. Traditionally, HPV genotypes, which are frequently identified in cervical neoplasms, have been segregated into those with a low risk and those with a high risk for malignant transformation. HPV types 6 and 11 are the prototypes of low-risk viruses that are most frequently found in low-grade squamous intraepithelial lesions (LSIL) and condylomata. HPV 16, 18, 31 , and 45 account for approximately $80 \%$ of invasive cervical carcinomas, and the HPV 16-related group is overrepresented in high-grade squamous intraepithelial lesions (HSIL) and squamous cancers compared with the HPV 18-related viruses. The expression of HPV mRNA and proteins provides a coherent model for cervical carcinogenesis (1-3).

Within the HPV genomes, the E6 and E7 genes are the driving forces behind epithelial proliferation and transformation. These transcribed genes are conserved and expressed within all HPV-infected pathologies. In many high-risk cervical dysplasias and invasive carcinomas, the E6 and E7 proteins are restrictively transcribed from the HPV genome integrated into the host DNA (4-6). The link between oncogenesis and the transforming properties of HPV infection was identified by the interaction between these proteins and host cellular tumor suppressor gene products. Specifically, E7 competitively binds to the retinoblastoma cellular tumor suppressor gene product (pRB), thereby increasing transcription of cell cycle proteins through release of the E2F transcription factor bound to pRB (7). The E7 protein of low-risk HPVs has a lower affinity 
for pRB and is less effective in this transactivation than the E7 derived from high-risk HPVs.

In addition to the transforming properties of E7, E6 binds to the wild type form of the p53 tumor suppressor gene product resulting in a ubiquitindependent proteolysis and degradation of p53. Resultant loss of wild type p53 increases cellular genomic instability after DNA damage. Somatic and germ line mutations of p53 have been identified in a wide variety of human carcinomas (8). However, in cervical cancers, this mutation is rarely detected (9-11). Thus, one concludes that E6-mediated p53 degradation as seen in cervical carcinoma results in the same oncogenetic effects as that seen with a p53 knockout mutation.

At least 10 p53 polymorphisms have been described in both coding and noncoding regions of the gene (12). A few polymorphic sites-in particular, one at codon 72 of exon 4 -have been studied extensively in regard to increased risk for cancer development. The codon 72 position has either an arginine (CGC) or proline (CCC) allele that seems to be functionally equivalent (13). Ethnic differences in these allelic frequencies exist as well (14). In the U.S. population, the proline allele is identified more commonly in African Americans (61\%) than in Caucasians (34\%) (13-15).

Polymorphisms at codon 72 have been studied in smoking- and non-smoking-related lung carcinomas with inconsistent findings. In some studies, patients with smoking-induced lung adenocarcinoma had a higher proline allele frequency. In contrast, in Japanese patients with lung carcinoma, proline homozygosity was not increased with any histologic type of lung cancer, but higher arginine homozygosity was seen in cancers that were not smoking related (16-20). It has been proposed that the codon 72 haplotype may represent a marker for linkage disequilibrium with a chromosome $17 \mathrm{p}$ gene that carries increased cancer development risk $(12,21)$.

In HPV-infected cervical carcinomas, Storey and colleagues found women homozygous for arginine at p53 codon 72 to be approximately seven times more likely to develop cancer than those who were heterozygous or homozygous for the proline allele (22). With the use of cell lines with transfected p53 and E6 expressing plasmids, E6-mediated degradation of p53 carrying the arginine allele was more effective than the degradation of the proline form of p53, regardless of whether the infection was with high- versus low-risk HPV types. This study was exciting in that it presented a logical argument that might explain neoplastic progression of HPVassociated tumors based, in part, on p53 phenotype.

However, there have been criticisms of Storey's study, including the lack of Hardy-Weinberg equilibrium in the control group, lack of ethnic homogeneity within the control group, a small sample size, and the quality of the DNA used for polymerase chain reaction (PCR) analysis. Interlaboratory variation in ascertaining p53 genotypes may lead to an underestimation or inability to detect an association. A number of independent studies since Storey's original findings have found no increased risk associated with arginine homozygosity (23-30) (Table 1). Studies involving European population samples (Norway, United Kingdom, Netherlands, Sweden, Germany, and France) of cervical carcinomas and/or squamous intraepithelial lesions (SILs) in Hardy-Weinberg equilibrium do not confirm Storey's findings, regardless of HPV status. Both leukocyte DNA and/or DNA extracted from tissues were used with similar results. In fact, in one study that used samples from Costa Rica, the Eastern United States, and Oregon, a decreased risk for cervical cancer was associated with p53 arginine homozygosity (25). In this study, we further investigate the

TABLE 1. Negative Studies on Codon 72 p53 Allelotype and Cervical Cancer Risk

\begin{tabular}{|c|c|c|c|c|c|c|c|}
\hline Author & Population & $\begin{array}{c}\# \\
\text { Control/ } \\
\text { LSIL }\end{array}$ & \# Cancer & \# HSIL & $\begin{array}{c}\text { DNA Source } \\
\text { Control/Cancer }\end{array}$ & $\begin{array}{c}\text { Storey } \\
\text { Amplicon }\end{array}$ & $\begin{array}{l}\text { Detection } \\
\text { Method }\end{array}$ \\
\hline Hayes & Netherlands & 158 & 25 & 147 & blood/blood & No & SSCP \\
\hline Helland & Norway & 334 & 77 & 92 & blood/blood & No(199bp) & RFLP \\
\hline \multirow[t]{3}{*}{ Hildesheim } & Costa Rica & 123 & 49 & 200 & blood/blood & No & SSCP \\
\hline & Portland & 217 & & 208 & $\mathrm{cvl} / \mathrm{cvl}$ & No & SSCP \\
\hline & Eastern US & 245 & 88 & 47 & $\mathrm{cvl} / \mathrm{cvl}$ & No & SSCP \\
\hline Josefsson & Sweden & 100 & 63 & 488 & $\begin{array}{l}\text { smear/formalin } \\
\text { fixed paraffin }\end{array}$ & No(164bp) & RFLP \\
\hline Klaes & Germany & 151 & 87 & & $\begin{array}{l}\text { swabs \& punch } \\
\text { biopsies }\end{array}$ & No(182bp) & RFLP \\
\hline Lanham & UK & 250 & 38 & 172 & & Yes & ETBR/gel \\
\hline Minaguchi & Japan & 110 & 103 & & blood/frozen & Yes & $32 \mathrm{P} /$ gel \\
\hline Riou & France/Algeria & 66 & 89 & & blood/? & No & RFLP \\
\hline Rosenthal & UK & 246 & 50 & & $\begin{array}{l}\text { blood/formalin } \\
\text { fixed paraffin }\end{array}$ & Yes & ETBR/gel \\
\hline
\end{tabular}

LSIL, low-grade squamous intraepithelial lesion; HSIL, high-grade squamous intraepithelial lesion; SSCP, single-strand conformation polymorphism; RFLP, restriction fragment length polymorphism; ETBR, ethidium bromide. 
linkage of p53 arginine homozygosity in the spectrum of cervical neoplasia using a variation of Storey's method, as well as a restriction fragment length polymorphism (RFLP)-based method applied to DNA extracted from paraffin sections under microscopic control.

\section{MATERIALS AND METHODS}

Paraffin blocks of cervical pathologies were derived from the archives of the Department of $\mathrm{Pa}$ thology at the University of Virginia Health Sciences Center. In addition, some cases were derived from studies reported previously in a survey of HPV typing of cervical lesions (2). Paraffin sections were serially cut with the final sections stained with hematoxylin and eosin to morphologically control the presence of lesion tissue within each block. In the vast majority of cases, the samples were derived from conization specimens, thereby ensuring that the diagnosis was accurate compared with studies that used colposcopic biopsies as a standard. Samples included 123 LSILs, 148 HSILs, 102 invasive cervical squamous carcinomas including 78 large cell nonkeratinizing and 24 keratinizing types. In addition, 32 cases of adenocarcinoma in situ (ACIS) and 28 invasive adenocarcinomas of various grades and 3 small cell neuroendocrine cancers were studied, for a total of 431 evaluable cases. A case was defined as evaluable if lesion tissue was still available for microdissection and if the tissue was found to be amplifiable based on control PCR amplification as previously described (31). Controls included adequate amplification for $\beta$-globin and coincidentally processed HPV containing cancer cell lines known to be homozygous or heterozygous at this locus, as well as numerous negative controls. The method of Storey et al. was adapted as follows: The exact primer sets used for codon 72 differential amplification as described in that article were used (22). Amplification cycling conditions were optimized to include activation of the AmpliTaq Gold polymerase (Perkin-Elmer, Norwalk, CT) at $94^{\circ} \mathrm{C}$ for $13 \mathrm{~min}$ followed by 50 cycles of 30 seconds each at $94^{\circ} \mathrm{C}, 58^{\circ} \mathrm{C}$, and $72^{\circ} \mathrm{C}$, respectively. At the end of the 50 cycles, there was a 10 -min extension time at $72^{\circ} \mathrm{C}$. The proline and arginine primer sets were found to amplify optimally with a final magnesium concentration of $1.5 \mathrm{~mm}$. For $\beta$-globin amplification, a magnesium concentration of $3.5 \mathrm{~mm}$ was used. Furthermore, formamide was added to a final concentration of $1 \%$ to optimize further the amplification in all three reactions. The proline and arginine primers were used at a final dilution of 100 $\mathrm{nm}$, and $\beta$-globin primers were used at $40 \mathrm{~nm}$. After amplification (20\%) of the $50 \mu$ l reaction was run on an agarose gel and the fractionated products were visualized after staining with $10 \mu \mathrm{g} / \mathrm{mL}$ ethidium bromide using an AlphaImager (Alpha Innotech, San Leandro, CA) apparatus.

RFLPs for codon 72 of p53 were also analyzed using the method of Klaes et al. (27). This was applied to all samples from the study, which were adequate for amplification but were negative for the proline allele by the Storey method. Primers as described in Klaes et al. that generated a 182-base pair fragment containing the polymorphic p53 codon 72 were used in a 50-cycle PCR. This was optimized with $2 \mathrm{~mm} \mathrm{MgCl}_{2}, 67 \mathrm{~nm}$ primer concentrations, $1 \%$ formamide with a $62^{\circ} \mathrm{C}$ annealing temperature, all other parameters being identical to the previously described PCR. Twenty percent of the amplified product was subjected to restriction enzyme digestion with BstU1 endonuclease at $60^{\circ} \mathrm{C}$ for $18 \mathrm{~h}$. The resulting fragments were visualized on $3 \%$ agarose gels and stained with $10 \mu \mathrm{g} / \mathrm{mL}$ ethidium bromide. The RFLP patterns as described in Klaes et al. are as follows: Homozygous proline DNA generates a single 182-base pair undigested fragment; a homozygous arginine allele generates 133-base pair and 49-base pair fragments. Heterozygotes generate mixtures of all three bands. To cross-check these results, any questionable or discrepant cases were subjected to additional testing using the restriction endonuclease BsaJ1, also as described in Klaes et al.

Comparisons in the allelic frequencies of the p53 polymorphisms at codon 72 as determined by the Storey method versus the Klaes method were carried out using Bowker's test for correlated proportions (32). The risk for cancer attributable to arginine homozygosity by each allelic classification method was assessed using logistic regression analysis. $\chi^{2}$ tests of contingency tables were used to assess differences in allelic proportions between the diagnostic classes within a single allelic classification method. The SAS System for Windows, version 6.11 (SAS Institute, Cary, NC) was used throughout for these statistical analyses.

\section{RESULTS}

Results of the above analyses are tabulated in Table 2. Of these 431 cases, 29 had indeterminate results with the use of the modified Storey method alone. This was most often the result of failure of amplification of both p53 alleles or a weak/indeterminate $\beta$-globin band on the gels. In contrast, when these indeterminate cases were further analyzed using the RFLP method, only eight cases were found to be uninterpretable. Thus, 423 of 431 cases could be assigned a final p53 phenotype using DNA extracted from the paraffin sections that had their morphologic content confirmed. Of these 423 


\begin{tabular}{|c|c|c|c|c|c|c|}
\hline Diagnosis & $\begin{array}{l}\text { Total } \\
\text { Cases }\end{array}$ & $\begin{array}{l}\text { p53 } \\
\text { Eval. }\end{array}$ & $\begin{array}{c}\text { \# HO } \\
\text { PRO (\%) }\end{array}$ & $\begin{array}{c}\text { \# HO ARG } \\
(\%)\end{array}$ & $\begin{array}{c}\text { \# HET } \\
(\%)\end{array}$ & Indet \\
\hline \multicolumn{7}{|l|}{ Combined Method } \\
\hline LSIL & 123 & 121 & $16(13.2)$ & $54(44.6)$ & $51(42.1)$ & 2 \\
\hline HSIL & 143 & 143 & $13(9.1)$ & $66(46.1)$ & $64(44.8)$ & 0 \\
\hline ACIS & 32 & 32 & $5(15.6)$ & $14(43.8)$ & $13(40.6)$ & 0 \\
\hline Invasive & 133 & 127 & $13(10.2)$ & $58(45.7)$ & $56(44.1)$ & 6 \\
\hline HSIL+ACIS & 175 & 175 & $18(10.3)$ & $80(45.7)$ & $77(44)$ & 0 \\
\hline \multicolumn{7}{|c|}{ Modified Storey Method } \\
\hline LSIL & 123 & 115 & $13(11.3)$ & $64(55.7)$ & $38(33)$ & 8 \\
\hline HSIL & 143 & 141 & $11(7.8)$ & $84(59.6)$ & $46(32.6)$ & 2 \\
\hline ACIS & 32 & 31 & $6(19.4)$ & $21(67.7)$ & 4 (12.9) & 1 \\
\hline Invasive & 133 & 115 & $7(6.1)$ & $77(67)$ & $31(27)$ & 18 \\
\hline HSIL+ACIS & 175 & 172 & 17 (9.9) & $105(61)$ & 50 (29) & 3 \\
\hline
\end{tabular}

HO, homozygous; PRO, proline; ARG, arginine; HET, heterozygous; Indet, indeterminate.

cases, the frequency of arginine homozygosity ranged from 43.8 to $46.1 \%$. All diagnostic subgroups were found to be in Hardy-Weinberg equilibrium. $\chi^{2}$ analysis failed to reveal any statistically significant difference among the allelic proportions in each diagnostic class. Subgroup analyses of subtypes of invasive carcinoma, as well as grouping all preinvasive high-grade lesions versus invasive cancers, also failed to reveal any significant difference.

In contrast, there was a highly statistically significant difference in allelic frequencies when the modified Storey method was compared with the final phenotype using RFLP confirmation. With the Storey method alone, the frequency of arginine homozygosity ranged from $55.7 \%$ in low-grade lesions to $67 \%$ for invasive carcinomas. This trend suggesting a correlation between invasive disease and arginine homozygosity, which is similar to that reported by Storey et al., failed to reach statistical significance. Analysis of the primary data raised a concern that the amplifiability of the two amplicons generated in this method was not equivalent, despite optimization of protocols. Most frequently, this was represented as a weaker proline band compared with the arginine band seen on the gels (Fig. 1). This could not be explained by loss of heterozygosity given the large amount of control normal tissue present in virtually all specimens. Hence, we chose to verify all apparent arginine homozygotes using the RFLP method, as well as an appropriate number of randomly selected control cases. When results were compared and a final p53 phenotype was assigned, the apparent trends among the cases disappeared (Fig. 2). With the use of logistic regression analysis and grouping cases into cancer versus noncancer, the odds ratio based on the final phenotype was 1.00 for the development of cancer, clearly not statistically significant $(P=.9952)$. However, with the use of the modified Storey method alone and similarly grouping the cases, the odds ratio was 1.42 with a $P$ value of 0.134 , suggesting a trend but still not statistically significant. In the analysis of proportions, all of the transitions were in

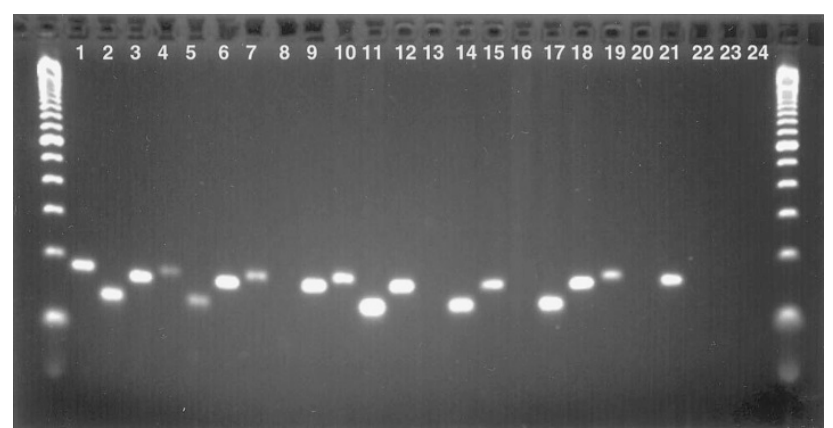

FIGURE 1. Representative data using the modified Storey method as listed in the text. Each triplet represents amplification of the proline, arginine, and $\beta$-globin amplicons, respectively. At the end of the gel are 100 base-pair molecular weight ladders. Lanes 1-9 demonstrate data from human papillomavirus (HPV)-containing cervical cancer cell lines CaSki (HPV 16; known heterozygote), Hela (HPV 18; known heterozygote), and Siha (HPV 16; known proline homozygote). Lanes 10-12 contain DNA from a heterozygous squamous cell carcinoma. Lanes 13-15 contain DNA from a homozygous squamous cell carcinoma. Lanes 16-18 contain DNA from a squamous cell carcinoma apparently homozygous for arginine by this method. Lanes 19-21 represent DNA from a low-grade squamous intraepithelial lesion that is homozygous for proline. Lanes 22-24 are negative controls.

the same direction for all group and subgroup analyses. Transitions were always from apparent arginine homozygosity to final phenotype of heterozygosity with the overall $P$ value for the comparison between the two methods of 0.001 (Bowker's test). Thus, the statistical analysis supports a seeming methodologic difference. These findings help explain the variable reports in the literature and strongly suggest that inadequate sample size determination and differing methodologies explain the variability in results.

\section{DISCUSSION}

Alterations of p53, either through genetic mutation or allelic loss, have been described as the most common genetic changes in human cancers, including those of mammary, pulmonary, and colonic origin (33). In HPV-associated neoplasms, these genetic alterations are usually absent but functionally present because of E6-mediated degradation of p53. Recent 


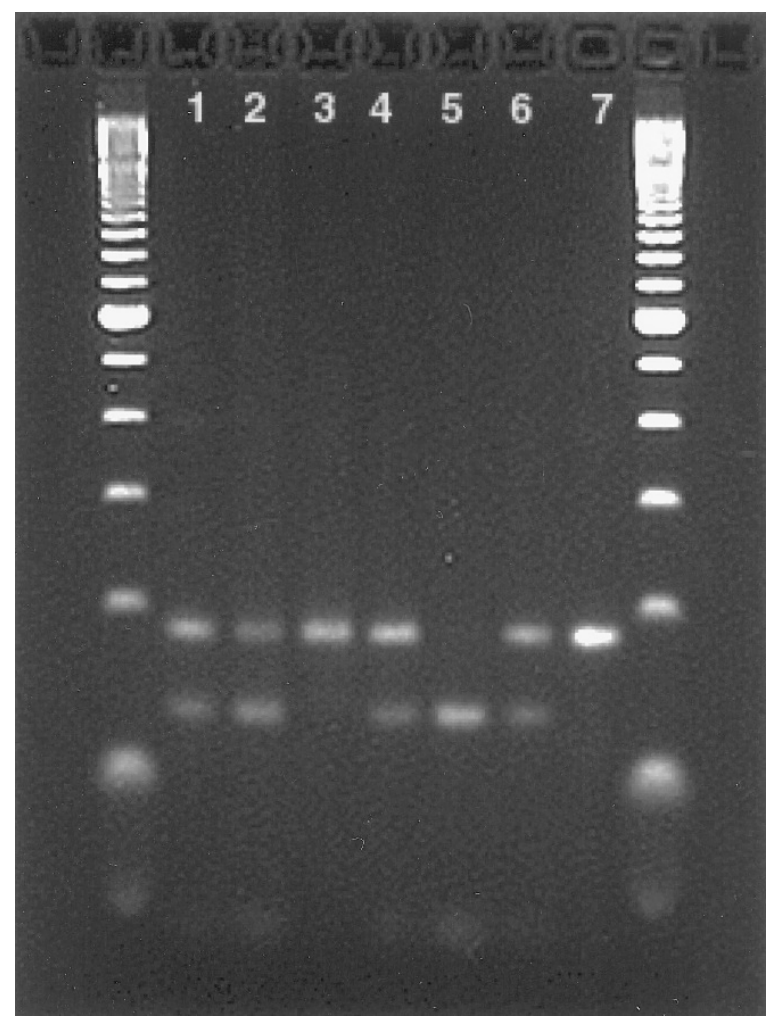

FIGURE 2. Restriction fragment length polymorphism analysis via the method of Klaes et al. (27) for the cases represented in Figure 1. Lane 1, CaSki. Lane 2, Hela. Lane 3, Siha. Lanes 4-7, the four cases represented in lanes 13-21, respectively, of Figure 1. Note: Lane 6 demonstrates heterozygosity for the apparently homozygous arginine result in lanes 16-18 of Figure 1. In contrast, lanes 5 (homozygous arginine) and 7 (homozygous proline) confirm the previous results, as do all controls.

literature has considered p53 allelic polymorphisms to be possible predisposing factors for tumor development. These changes in conjunction with certain environmental exposures (e.g., smoking, lung cancer) may increase the risk for cancer development in people with susceptible genotypes. However, these studies have been inconsistent, most likely because of a number of factors, including racial frequency differences between cases and controls, varied population bases, and lack of Hardy-Weinberg equilibrium in some examined cases (15-20). In our opinion, these variables and others account for the discrepant results between independent studies examining the polymorphism of p53 at codon 72 and carcinogenesis.

Storey and colleagues reported a tantalizing 7-fold increased risk for cervical carcinoma associated with p53 arginine homozygosity at codon 72 (22). The cell line data described in that study provided a molecular rationale to support this clinical observation. However, this study has been criticized, primarily because of its small sample size, consisting of 41 controls and 30 cancers. For example, Rosenthal et al., using Storey's method, analyzed a UK population of 246 controls and 50 cervical cancers with DNA extracted from blood (control) and archival tissue (cancer) and found no difference in polymorphism frequency (30). Other criticisms have included uncertain ethnicity of the cases with a lack of Hardy-Weinberg equilibrium in the control group. Storey and colleagues' arginine homozygosity frequency in the control group is lower (37\%) than the frequency reported in other European studies of 57 or $63 \%(27,30)$. However, it is similar to that of Minaguchi et al.'s Japanese study that did not find a correlation between arginine homozygosity and cancer (29). Furthermore, the tissue used for DNA extraction differs between cancer and control groups (frozen tissue for cancer versus whole blood leukocyte DNA for controls). Whether any difference in DNA quality between these two preparation methods affected results using Storey's PCR methodology has also been questioned (26).

Stimulated by Storey's provocative results, multiple studies, as listed in Table 1 have found no increased risk for cervical cancer associated with arginine homozygosity. Most of these studies used a larger number of cases with better-defined ethnic population bases and meet Hardy-Weinberg equilibrium criteria. The exact methodology and DNA quality/source, however, varied quite a bit among the different studies. For example, Klaes et al. (27) analyzed a German population of 87 confirmed cervical cancers and 151 controls using punch biopsies or cervical cytobrush samples for DNA retrieval. This extracted DNA was amplified using a different exon 4 primer set than that used by Storey, and the resulting fragment was analyzed by RFLP using endonucleases BstU1 and BsaJ1. In our study, we used this same PCR/RFLP method as an alternative to our modified Storey method for all indeterminate cases and for confirmation of all arginine homozygotes. With the use of the modified Storey method alone, identified in that group was a trend toward arginine homozygosity that was not statistically significant. However, complimentary analysis confirmed this trend to be secondary to the relative weaker amplifiability of the proline amplicon used in the Storey method. By using an independent confirmation method, we were able to obtain an accurate phenotype of heterozygosity in many misclassified cases, and the trend disappeared. In comparison to most of the studies in Table 1, our study encompasses a broader range of histologically confirmed pathologies, including 165 carcinomas representing all of the most common histologic subtypes, 32 cases of ACIS, 143 HSILs, and 123 LSILs. The current study is the only report that has used two methods to investigate the codon 72 allelotype. Our study, as well as others that have incorporated various grades of preinvasive neoplasia, found no risk for progression associated with arginine homozygosity in any of these subgroups. 
In conclusion, with the use of a reasonably defined population base (mostly eastern United States, primarily Caucasian women), a much larger number of cases with all subgroups fitting HardyWeinberg equilibrium, uniformity of DNA quality/ source across all groups (formalin fixed conization specimens), and two separate methods for determination and confirmation of p53 allelotype, there is no increased cancer risk associated with arginine homozygosity. A trend toward arginine homozygosity was identified on the basis of the Storey method alone. However, our study demonstrates that with the use of an alternative method, this trend toward arginine homozygosity disappears and that this is most likely secondary to differential amplifiability of the primer sets used by Storey and colleagues. Clearly, both sample size and molecular methodologic variables account for many of the differences in the published literature on this topic.

\section{REFERENCES}

1. Stoler MH, Broker TR. In situ hybridization detection of human papillomavirus DNAs and messenger RNAs in genital condylomas and a cervical carcinoma. Hum Pathol 1986; 17(12):1250-8.

2. Stoler MH, Rhodes CR, Whitbeck A, Wolinsky SM, Chow LT, Broker TR. Human papillomavirus type 16 and 18 gene expression in cervical neoplasias. Hum Pathol 1992;23(2):117-28.

3. Stoler MH. The biology of papillomaviruses. Pathol Case Rev 1997;2(1):1-13.

4. Scheffner M, Werness BA, Huibregtse JM, Levine AJ, Howley PM. The E6 oncoprotein encoded by human papillomavirus types 16 and 18 promotes the degradation of p53. Cell 1990; 63(6):1129-36.

5. Scheffner M, Munger K, Byrne JC, Howley PM. The state of the p53 and retinoblastoma genes in human cervical carcinoma cell lines. Proc Natl Acad Sci U S A 1991;88(13):5523-7.

6. Werness BA, Levine AJ, Howley PM. Association of human papillomavirus types 16 and 18 E6 proteins with p53. Science 1990;248(4951):76-9.

7. Dyson N, Howley PM, Munger K, Harlow E. The human papilloma virus-16 E7 oncoprotein is able to bind to the retinoblastoma gene product. Science 1989;243(4893):934-7.

8. Law MR. Genetic predisposition to lung cancer. Br J Cancer 1990;61:195-206.

9. Crook T, Wrede D, Tidy JA, Mason WP, Evans DJ, Vousden $\mathrm{KH}$. Clonal p53 mutation in primary cervical cancer: association with human-papillomavirus-negative tumours [see comments]. Lancet 1992;339(8801):1070-3.

10. Fujita M, Inoue M, Tanizawa $\mathrm{O}$, Iwamoto S, Enomoto $\mathrm{T}$. Alterations of the p53 gene in human primary cervical carcinoma with and without human papillomavirus infection. Cancer Res 1992;52(19):5323-8.

11. Park DJ, Wilczynski SP, Paquette RL, Miller CW, Koeffler HP. P53 mutations in HPV-negative cervical carcinoma. Oncogene 1994;9(1):205-10.

12. Birgander R, Sjalander A, Rannug A, Alexandrie AK, Sundberg MI, Seidegard J, et al. P53 polymorphisms and haplotypes in lung cancer. Carcinogenesis 1995;16(9):2233-6.

13. Matlashewski GJ, Tuck S, Pim D, Lamb P, Schneider J, Crawford LV. Primary structure polymorphism at amino acid residue 72 of human p53. Mol Cell Biol 1987;7(2):961-3.

14. Beckman G, Birgander R, Sjalander A, Saha N, Holmberg PA, Kivela A, et al. Is p53 polymorphism maintained by natural selection? Hum Hered 1994;44(5):266-70.

15. Weston A, Godbold JH. Polymorphisms of H-ras-1 and p53 in breast cancer and lung cancer. Environ Health Perspect 1997;105:919-26.

16. Weston A, Perrin LS, Forrester K, Hoover RN, Trump BF, Harris CC, et al. Allelic frequency of a p53 polymorphism in human lung cancer. Cancer Epidemiol Biomarkers Prev 1992;1(6):481-3.

17. Weston A, Ling-Cawley HM, Caporaso NE, Bowman ED, Hoover RN, Trump BF, et al. Determination of the allelic frequencies of an L-myc and a p53 polymorphism in human lung cancer. Carcinogenesis 1994;15(4):583-7.

18. Kawajiri K, Nagachi K, Inai K, Wantanabe J, Hayashi S. Germ line polymorphisms of p53 and CYP1A1 genes involved in human lung cancer. Carcinogenesis 1993;14(6):1085-9.

19. Murata M, Tagawa M, Kimura M, Kimura H, Watanabe S, Saisho $\mathrm{H}$. Analysis of a germ line polymorphism of the p53 gene in lung cancer patients: discrete results with smoking history. Carcinogenesis 1996;17(2):261-4.

20. Murata M, Tagawa M, Kimura H, Kakisawa K, Shirasawa H, Fujisawa T. Correlation of the mutation of p53 gene and the polymorphism at codon 72 in smoking-related non-small cell lung cancer patients. Int J Oncol 1998;12(3):577-81.

21. Sjalander A, Birgander R, Kivela A, Beckman G. P53 polymorphisms and haplotypes in different ethnic groups. Hum Hered 1995;45:144-9.

22. Storey A, Thomas M, Kalita A, Harwood C, Gardiol D, Mantovani F, et al. Role of a p53 polymorphism in the development of human papillomavirus-associated cancer [see comments]. Nature 1998;393(6682):229-34.

23. Hayes VM, Hofstra RM, Buys CH, Hollema $\mathrm{H}$, van der Zee AG. Homozygous arginine-72 in wild type p53 and risk of cervical cancer [letter]. Lancet 1998;352(9142):1756.

24. Helland A, Langerod A, Johnsen H, Olsen AO, Skovlund E, Borresen-Dale AL. P53 polymorphism and risk of cervical cancer [letter; comment]. Nature 1998;396(6711):530-1; discussion 2.

25. Hildesheim A, Schiffman M, Brinton LA, Fraumeni JF Jr, Herrero R, Bratti MC, et al. P53 polymorphism and risk of cervical cancer [letter; comment]. Nature 1998;396(6711):531-2.

26. Josefsson AM, Magnusson PK, Ylitalo N, Quarforth-Tubbin P, Ponten J, Adami HO, et al. P53 polymorphism and risk of cervical cancer [letter; comment]. Nature 1998;396(6711): 531; discussion 2.

27. Klaes R, Ridder R, Schaefer U, Benner A, von Knebel Doeberitz M. No evidence of p53 allele-specific predisposition in human papillomavirus-associated cervical cancer. J Mol Med 1999;77(2):299-302.

28. Lanham S, Campbell I, Watt P, Gornall R. P53 polymorphism and risk of cervical cancer [letter; comment]. Lancet 1998; 352(9140):1631.

29. Minaguchi T, Kanamori Y, Matsushima M, Yoshikawa H, Taketani Y, Nakamura Y. No evidence of correlation between polymorphism at codon 72 of p53 and risk of cervical cancer in Japanese patients with human papillomavirus 16/18 infection [In Process Citation]. Cancer Res 1998;58(20):4585-6.

30. Rosenthal AN, Ryan A, Al-Jehani RM, Storey A, Harwood CA, Jacobs IJ. P53 codon 72 polymorphism and risk of cervical cancer in UK [see comments]. Lancet 1998;352(9131):871-2.

31. Gaffey MJ, Frierson HF, Weiss LM, Barber CM, Baber GB, Stoler MH. Human papillomavirus and Epstein-Barr virus in sinonasal Schneiderian papillomas: an in situ hybridization and polymerase chain reaction study. Am J Clin Pathol 1996; 106(4):475-82.

32. Dixon WJ, Masey FJ. Introduction to statistical analysis. New York: McGraw-Hill; 1983. p. 288-91.

33. Hollestein M, Sidransky D, Vogelstein B, Harris CC. P53 mutation in human cancers. Science 1991;253:49-53. 